\title{
論 文 Memoirs \\ On the Long Period Forecasting by Means of Harmonic Analysis (I)
}

\author{
K. Imahori and T. Kobayashi \\ (Research Irstitute of Low Temperature Science, Hokkaido University)
}

\section{Introduction}

The most systematic formulation which has ever been made of the theory of prediction of stationary time series is, to the best of the authors' knowledge, that of Kolmogoroff and Wiener ${ }^{(1)}$ which has recently been developed indepentdenly in U. S. S. R. and in U. S. A. Their theory is essentially a minimization problem in which is sought such a linear transformation that, when applied to the past and present values of a stationary time series, it will give the future values of the time series with as small errors as possible. It is mathematically rigorous, and covers a wide field of applications, so that it appears as if no room were left for any essentially new contributions except for possible extensions and applications following the lines established by the above mentioned authors.

Meanwhile, in the meteorological practice of weather forecasting there are two leading principles which characterize the various existing methods of forecasting. The one principle uses statistical methods, such as the correlation coefficient between the raifall at a particular district and the temperature of sea water at another. The method of periodogramme analysis may also be classified into this category. These statistical methods have a characteristic feature in that they can be used without any regard to possible physical mechanisms or causality relations between the quantities concerned. By the other principle of forecasting, on the other hand, one seeks for some physical law which governs the quantities entering into the phenomenon in question, and which may be effectively used for the purpose of prediction. The two principles are, of course, not independent. Various "theories" presented for the purpose of weather forecasting are approximate in sense that they cannot take all the variables into account which have some interconnection with the phenomenon under consideration, so that one must necessarily resort to the statistical method.

In the statistical formulation of the prediction problem, which might well be said to have been brought up to almost completeness by Kolmogoroff and Wiener, the physical bases or assumptions on which all the mathematical theories are built, and the physical meaning of various functions and formula occurring in them are apt to be left out of

(1) N. Wiener: Extrapolation, Interpolation, and Smoothing of Stationary Time Series, New York, 1949. 
consideration. Statistical treatments of, e.g., meteorological data may sometimes lead to deterministic physical laws in case of probability unity, but these are special cases of minor importance. In the theory of, e. g., Brownian motion, the observed irregular motion of a particle has a certain statistical regularity which may be expressed by the well-known Langevin equation, so that in general one might expect some physical law which, although unable to give precise prediction in a deterministic sense, expresses the interrelation or the mechanism existing in the phenomenon under consideration and enables one to draw out conclusions as to the effect produced under the given conditions.

In study on the statistical analysis of brain-waves, one of the present authors and Dr. K. Suhara (3) have formulated a theory in which is songht such a linear operator that, when operated on the observed brain-wave, this wave may be transformed into a completely random time series. The operational equation constructed in this way is, in the case of Brownian motion, reduced to the Langevin equation, and thus it is regarded in general as a tentative physical law in the above sense. While in the case of brain-waves nothing is known at present as to the mechanism of generation, so that a purely statistical attack has been the only one available for any systematic fo:mulation, there are many examples in which informations from different sources can be utilized in assuming a physical model which is governed by some known physical law. In view of the most effective application of the statistical theory to meteorological forecasting, the most interesting problem is how to combine these two methods of attack into a single formulation.

Although some methodological consideration on this problem has been made by one of the present authors in another field of study, ${ }^{(3)}$ the application of the same method to meteorological forecasting was not put into practice until last summer Dr. K. Takahashi of the Meteorological Research Institute visited sapporo and held a lecture on the method of periodogram analysis applied to his researches on seasonal forecasting. When the authors' theory was formulated to a certain extent and some numerical results were obtained as to the probable temperature of the coming winter at Sapporo, the authors were made aware of the above mentioned works of Kolmogoroff and Wiener. The present paper is a revised formulation of the manuscript prepared for presenting to the annual meeting of the Japanese Meteorological Society held in Nov. 1950. The authors do not pretend to have given a completed theory, but it is hoped that their contribution may add something to the development of the prediction theory as a physical science.

\section{Fundamental Assumptions}

Let the quantities which are used to describe the state of the system under consideration be expressed by functions $x(t, \alpha)$ 's of time $t$ in which a parameter $\alpha$, assuming a continuous or discrete set of values, is usad to distinguish different quantities. In case the

(2) K. Imahori and K. Suhara: Folia Psych. et Neul. Jap., Vol. 3, No. 2, 137, I949.

(3) K. Imahori: Bulletin of the Res. Ins. Applied Elect., Vol. 1, No. 1, 1949. 
variables $x$ 's depend in a completely definite way on the independent variable $t, x(t, \alpha)$ 's are said to be causal processes, and the procedure by which they are determined from a set of given conditions may be formulated as follows.

A system of finite or enumerably infinite number of functions $q_{1}(t), q_{2}(t), \ldots, q_{n}(t)$ is introduced, which are derived from $x(t, \alpha)$ 's by a set of transformations

$$
q_{i}(t)=K_{i}\{x(t, \alpha)\}, \quad i=1,2, \ldots, n,
$$

where $K_{1}, K_{2}, \ldots, K_{n}$ are operators which transform the function $x(t, \alpha)$ into $q_{1}(t), q_{2}(t)$, $\ldots, q_{n}(t)$ respectively. The $n$-dimensional space defined by the variables $q_{1}, q_{2}, \ldots, q_{n}$ may be used to represent the possible states of the system, and is called the phase space of the system. Starting from a point $q_{20}, q_{20}, \ldots, q_{n 0}$ on which the system finds itself at a particular time $t=0$, one may successively follow the path of the representiative point as time proceeds, provided that the limit of the rate of change in coordinates in a small time linterval $\Delta t$ exists for $\Delta t \rightarrow 0$, and is defined as a single valued function of coordinates, $^{(4)}$ i. e.,

$$
\frac{d q_{i}}{d t}-F_{i}(q)=0, \quad i=1,2, \ldots, n
$$

The problem is thus reduced to the solution of these simultaneous differential equations under the given initial conditions. In dynamical systems they correspond to the equations of motion expressed in Hamilton's canonical form, and the functional forms of $F_{1}, F_{2}, \ldots \ldots$, $F_{n}$ are determined by the dynamical structure of the system. In the present case the equations (2) are also called equations of motion of the system, and the functions $F_{i}$ 's are regarded to be characteristic of the system considered. The number of dimensions $n$ should also be characteristic of the system in order that the above requirement of unique determination of the process is to be fulfilled, while it is to a certain extent a matter of convenience what kind of transformations introduced in (1) should be adopted. Linear transformations are generally used, for example,

or

$$
\begin{aligned}
& q_{\varepsilon}(t)=x, \frac{d x}{d t}, \frac{d^{2} x}{d t^{2}}, \ldots, y, \frac{d y}{d t} \text {. etc., } \\
& q_{t}(t)=x(t), x(t+\tau), x(t+2 \tau), \ldots, y(t), y(t+\tau), \text { etc. }
\end{aligned}
$$

So much for the causal process. - A random process is not a causal process, so that the variables are not determined uniquely as functions of the time, the only available information being their probability distributions when the measurement is repeated sufficiently many times. Using the same transformations (1), the increments $\Delta_{q_{i}}$ of each variable in a short time $\Delta t$ are distributed according to some probability law. This may be expressed

(4) The more general case where the functions $F_{i}$ 's contain time explicitly is not considered here although the generalization might not be very difficult. In equation (2) $q$ stands for $q_{1}, q_{2}$, $\ldots . . ., g_{n}$, the same convention will be frequently used throughout this paper. 
by such conditional probability function depending upon the coordinates $q_{t}, q_{i}{ }^{\prime}=q_{t}+\Delta q_{t}$. and the time interval $\Delta t^{(5)}$, that when the coordinates are known to be $q_{1}, q_{2}, \ldots, q_{i b}$ at time $t$, the probability that they lie between $q^{\prime}, q_{2}{ }^{\prime}, \ldots, q_{n}{ }^{\prime}$ and $q_{1}{ }^{\prime}+d q_{1}{ }^{\prime}, \ldots, q_{n^{\prime}}{ }^{\prime}+d q_{n^{\prime}}{ }^{\prime}$ at time $t+\Delta t$ is given by

$$
P\left(q, q^{\prime}, \Delta t\right) d q^{\prime}
$$

Here is involved the assumption that the process is a Markoff process in which the dependence of the distribution function on the coordinates is restricted only to the initial coordinates, whatever the history previous to it may be. The plausibility of this assump-tion might be seen in the similar situation as stated in the case of causal processes.

Using (5), the first and second moments of the changes in the coordinates in a small: time interval $\Delta t$ are given by

$$
\left.\begin{array}{c}
a_{t}(q, \Delta t)=\int \ldots \int\left(q^{\prime}-q_{i}\right) P\left(q, q^{\prime}, \Delta t\right) d q^{\prime}, \\
b_{i j}(q, \Delta t)=\int \cdots \int\left(q_{i}^{\prime}-q_{i}\right)\left(q_{i}^{\prime}-q_{i}\right) P\left(q, q^{\prime}, \Delta t\right) d q^{\prime}, \\
i, j=1,2, \ldots, n .
\end{array}\right\}
$$

It is assumed that in the limit $\Delta_{t} \rightarrow 0$, all the $a_{i}$ 's and $b_{t j}$ 's become proportional to $\Delta t_{z}$ sothat the equations

$$
\begin{aligned}
& A_{i}(q)=\lim _{\Delta t \rightarrow 0} \frac{a_{i}(q, \Delta t)}{\Delta t} . \\
& B_{i j}(q)=\lim _{\Delta t \rightarrow 0} \frac{b_{t 1}(q, \Delta t)}{\Delta t} . \\
& i, j=1,2, \ldots, n
\end{aligned}
$$

exist. Then it can be shown that a generalized Fokker-Planck equation

$$
\frac{\partial P}{\partial t}=-\sum_{i=1}^{n} \frac{\partial}{\partial q_{l}}\left[A_{i}(q) P\right]+\frac{1}{2} \sum_{k, l} \frac{\partial^{2}}{\partial q_{k} \partial q_{l}}\left[B_{k l}(q) P\right]
$$

holds, where $P$ is regarded as a function of $q_{1}, q_{2}, \ldots, q_{n}$ and $t$, and the initial values of coordinates are contained as parameters. Thus, if the functional forms of $A_{i}$ 's and $B_{\xi}$ 's are assumed to be known, the problem is reduced to the solution of the diffusion equation (8) under the initial condition:

$$
P\left(q_{1}, q_{2}, \ldots, q_{n}, 0\right)=\delta\left(q_{1}-q_{10}, \ldots, q_{n}-q_{n 0}\right),
$$

where $\delta(q)$ is so-called Dirac's $\delta$-function.

The direct physical meaning of the functions $A_{i}$ 's and $B_{i}$ 's is obvious from theirdefinitions, but another interesting interpretation may be obtained in connection with a

(5) The dependence upon the absolute position in time is also left out; of consideration, if ifoot note on page 367 . 
possible physical law by which the change in time of the coordinates may be described: While the mean rate of change of the coordinate $q_{6}$ is given by $A_{i}(q)$, the actual rate of change $d q_{i} / d t$ will differ from it by a quantity which is totally unpredictable, so that one may write

$$
\frac{d q_{i}}{d t}-A_{i}(q)=p_{i}(t), \quad i=1,2, \ldots, n,
$$

in which $p_{t}$ 's as functions of $t$ have the following properties:

$$
\left.\begin{array}{rlrl}
\overline{p_{i}(t)} & =0, & i=1,2, \ldots, n \\
\overline{p_{i}\left(t^{\prime}\right) p_{j}\left(t^{\prime \prime}\right)}=B_{t j} \delta\left(t^{\prime}-t^{\prime \prime}\right), & i, j=1,2, \ldots, n
\end{array}\right\}
$$

To use in the second equation the same notation $B_{6}$, as in (7) is justified by calculating the second moments. Thus from (10) one gets

$$
\begin{gathered}
\Delta q_{i}=A_{i}(q) \Delta t+\int_{0}^{\Delta t} p_{i}(t) d t \\
\overline{\left.\Delta q_{i} \Delta_{q}\right)}=A_{i}(q) A i(q)(\Delta t)^{2}+\iint_{0}^{\Delta t} \overline{p_{i}\left(t^{\prime}\right) p_{j}\left(t^{\prime \prime}\right)} d t^{\prime} d t^{\prime \prime}, \\
\lim _{\Delta t \rightarrow 0} \frac{\overline{\Delta q_{t} \Delta q_{i}}}{\Delta t}=B_{t}
\end{gathered}
$$

wheich is the result to be shown.

It is interesting to note that the equation (10) may be regarded as a generalization of the equation (2), the functions $A_{i}(q)$ of the former corresponding to the functions $F_{i}(q)$ of the latter, and that the functions $p_{i}(t)$ resemble the external random "forces" in the case of Brownian motion. They play the same role as the so-called Langevin equations in the theory of Brownian motion, and thus can be regarded as representing a possible physical model of the system.

\section{Theory of Linear Prediction}

The differential equations (10) of the preceding article cannot always be considered as linear, because one has no a priori knowledge as to the reason why the functional form of $A_{i}(q)$ should assume some particular structure except when this is given or assumed, at least, from the known structure of the system in question. It is not easy to give simple examples in which the phenomena are governed by'non-linear laws, so that their complete formulations are not yet obtained. It seems, however, to exist one way of getting rid of this difficulty. The key point is that a statistical ensemble of any physical systems, linear or non-linear, might be considered as equivalent to that of appropriately chosen linear systems. Some considerations along this line are now being made, but the details will not be described here, and the present report will deal only with the case where a liuear law can be assumed to exist. 
Assuming that $A_{s}(q)$ is linear in coordinates, one puts

$$
A_{i}(q)=\sum_{j=1}^{n} a_{i, j} q_{i}, \quad i=1,2, \ldots, n,
$$

so that the equation (10) becomes

$$
\frac{d q_{i}}{d t}-\sum_{j} \alpha_{i j} q_{j}=p_{i}(t), \quad i=1,2, \ldots, n,
$$

where the coefficients $a_{t, j}$ 's are now considered as constants characteristic to the system. The corresponding Fokker-Planck equation may be written

$$
\frac{\partial P}{\partial t}=-\sum_{i j} a_{i j} \frac{\partial}{\partial q_{t}}\left[q_{j} P\right]+\frac{1}{2} \sum_{i j} B_{i j} \frac{\partial^{2} P}{\partial q_{i} \partial q_{j}}
$$

The solution of (12) or (13) can be obtained in various forms. It is convenient to begin with an orthogonal transiormation defined by

$$
z_{i}=\sum_{j=1}^{n} c_{i j} q_{j,} \quad i=1,2, \ldots, n,
$$

such that

$$
\sum_{j} c_{i}, a_{i k}=\lambda_{i} c_{i k}, \quad i, k=1,2, \ldots, n
$$

where $\lambda_{i}$ 's are solutions of a determinantal equation

$$
\text { Det. }\left(a_{i j}-\lambda \delta_{i}\right)=0
$$

Then the differential equation will be reduced to

$$
\frac{d z_{t}}{d t}-\lambda_{i} z_{i}=\pi_{i}(t), \quad i=1,2, \ldots, n
$$

where

$$
\pi_{i}(t)=\sum_{j} c_{4 j} p_{j}(t)
$$

so that

$$
\left.\overline{\pi_{i}\left(t^{\prime}\right)} \overline{\pi_{i}\left(t^{\prime \prime}\right.}\right)=\sigma_{i j} \delta\left(t^{\prime}-t^{\prime \prime}\right)=\sum_{k, t} c_{i k} c_{j l} B_{k: l} \delta\left(t^{\prime}-t^{\prime \prime}\right),
$$

and the Fokker-Planck equation becomes

$$
\frac{\partial P}{\partial t}=-\sum_{i} \lambda_{i} \frac{\partial}{\partial z_{i}}\left[z_{i} P\right]+\frac{1}{2} \sum_{i j} \sigma_{i j} \frac{\partial^{2} P}{\partial z_{i} \partial z_{j}} .
$$

The solution of the last equation was given by Ming Chen Wang and G. E. Uhlenbeck ${ }^{(6)}$ as follows:

$$
F(\xi, t)=\operatorname{Exp} .\left[-i \sum_{j} \xi_{j j_{j 0}} e^{\lambda_{j} t}+\frac{1}{2} \sum_{j k} \sigma_{i k} \frac{\xi_{j} \xi_{k}}{\lambda_{j}+\lambda_{k}}\left\{1-e^{\left(\lambda_{j}+\lambda_{k}\right) t_{j}}\right\}\right],
$$

where $F(\xi, t)$ is the Fourier transform of $P(z, t)$, and $z_{j 0}$ the initial value of $z_{j \cdot}$. The probability function $P(z, t)$ is thus a $n$-dimensional Gausssian distribution with the average value

and the variances

$$
\bar{z}_{i}=z_{\tau_{0}} e^{\lambda_{i} t}, \quad i=1,2, \ldots, n
$$

(6) Ming Chen Wang and G. E. Ublenbeck: Rev. Mod. Phys., 17, 323. 1935. 


$$
\overline{\left(z_{i}-\overline{z_{i}}\right)\left(z_{j}-\overline{z_{j}}\right)}=-\frac{\sigma_{i 1}}{\lambda_{b}+\lambda,}\left[1-e^{\left(\lambda_{0}+\lambda_{j}\right) t}\right], \quad i, j=1,2, \ldots, n
$$

The solution of the Langevin equation (17) can also be obtained easily:

$$
\begin{gathered}
z_{i}(t)=\int_{0}^{\infty} \pi_{i}\left(t-t^{\prime}\right) e^{\lambda_{i} t^{\prime}} d t^{\prime}=\int_{-\infty}^{t} \pi_{i}\left(t^{\prime}\right) e^{\lambda_{i}\left(t-t^{\prime}\right)} d t^{\prime}, \\
i=1,2, \ldots, n .
\end{gathered}
$$

It is interesting to note that the auto- and cross-correlation functions for $\boldsymbol{z}_{i}$ 's are intimately related to the above expressions (22) and (23), which characterize the probability function. One gets from (24)

$$
\begin{aligned}
& Z_{i j}(\tau)=\left[z_{i}(t+\tau) z_{j}(t)\right]_{t}=\int_{0}^{\infty} \int_{0}^{\infty}\left[\pi_{i}\left(t+\tau-t^{\prime}\right) \pi_{i}\left(t-t^{\prime \prime}\right)\right]_{t} e^{\lambda_{i} t^{\prime}+\lambda_{j} t^{\prime \prime}} d t^{\prime} d t^{\prime \prime} \\
& = \begin{cases}\frac{\sigma_{i j}}{\lambda_{i}+\lambda_{j}} e^{-\lambda_{j} \tau}, & \text { for } \tau<0, \\
\frac{\sigma_{i j}}{\lambda_{i}+\lambda_{j}} e^{\lambda_{i} \tau}, & \text { for } \tau>0,\end{cases}
\end{aligned}
$$

in which it is assumed that the real parts of $\lambda_{i}$ 's are negative, and [ $]_{t}$ means the average with respect to $t$. Thus the function $Z_{i f}(t)$ satisfies the differential equation

$$
\frac{d Z_{i j}}{d t}=\lambda_{i} Z_{i}, \quad \text { for } t>0 \text {, }
$$

which is obtained by equating the right hand side of (17) equal to zero. This property can be extended to more general cases in which only the linearity is assumed for the Langevin equation. Note that the average "motion" given by the equation (22) has also the same property.

From the above deseription it is seen that the problem of prediction has been essentially solved. The initial coordinates $z_{\imath 0}$ 's given, the average value of $z$ 's and the variances at the time later by $t$ can be calculated from ( $(2)$ and (23), provided that $\lambda_{i}$ 's are known. Transforming back to the original coordinates, one obtains the following expressions;

$$
\begin{aligned}
& P=\frac{1}{(2 \pi)^{n / 2} \Delta^{1 / 2}} \operatorname{Exp} \cdot\left[-\frac{1}{2} \sum_{i j} \tilde{D}_{i j}\left(q_{i}-\bar{q}_{t}\right)\left(q_{j}-\bar{q}_{j}\right)\right], \\
& \Delta=\operatorname{Det} .\left(D_{i j}\right), \quad\left(\tilde{D}_{t j}\right)=\left(D_{i j}\right)^{-1}, \\
& \bar{q}_{i}=\sum_{j k .} \tilde{c}_{i j} c_{j k} q_{k 0} e^{\lambda j t}, \quad\left(\tilde{c}_{i j}\right)=\left(c_{i j}\right)^{-1} \\
& \left.\overline{\left(q_{l}-\bar{q}_{i}\right)\left(q_{j}-\bar{q}_{i}\right)}=D_{l}\right)=-\sum_{k l} \tilde{c}_{i k} \tilde{c}_{j l} \frac{\sigma_{k l}}{\lambda_{k i}+\lambda_{l}}\left[1-e^{\left(\lambda_{k}+\lambda_{l}\right) l}\right], \\
& q_{t}(t)=\sum_{j} \tilde{c}_{i j} \int_{0}^{\infty} \pi_{j}\left(t-t^{\prime}\right) e^{\lambda t^{\prime}} d t^{\prime}=\sum_{j} \tilde{c}_{i} \int_{-\infty}^{t} \pi_{j}\left(t^{\prime}\right) e^{\lambda_{j}\left(t-t^{\prime}\right)} d t^{\prime}, \\
& -7-
\end{aligned}
$$




$$
\begin{aligned}
& Q_{t}(\tau)=\left[q_{\imath}(t+\tau) q_{j}(t)\right]_{i} \\
& =\left\{\begin{array}{ll}
\sum_{k l} \frac{\tilde{e}_{i k} \tilde{e}_{l l} \sigma_{k l}}{\lambda_{k}+\lambda_{l}} e^{-\lambda_{l} \tau}, & \text { for } \tau<0 \\
\sum_{k l} \frac{\tilde{e}_{l k} \tilde{e}_{l l} \sigma_{k l}}{\lambda_{k l}+\lambda_{l}} e^{\lambda_{h} \tau}, & \text { for } \tau>0
\end{array}\right\} \\
& \frac{d Q_{i j}}{d t}-\sum_{i k} a_{i k} Q_{k j}=0, \quad \text { for } t>0 \text {. }
\end{aligned}
$$

The last equation may be utilized to determine the $n^{2}$ constants $a_{6 j}$ 's from the experimentally obtainable functions $Q_{k i}$ 's, so that by solving (15) and (16) one obtains the coefficients $e_{t}$ 's of diagonal transformation and the principal values $\lambda_{\xi}$ 's. The "diffusion" constants $\sigma_{i}$ 's are determined from (29).

The formal procedure of prediction sketched above will bo seen to be in agreement with that used by Mr. Ogawara in some of the applications of his theory of stochastic extrapolation. (7) In practical applications of the above theory, however, one is aure to be perplexed by the calculations involving high order determinants, for in most cases the order $n$ of the determinants must be taken so large that neither the evaluation of these determinants nor the solution of the determinantal equation (16) can be carried on. While Mr. Ogawara assumed a very small number of dimensions ( $n=$ from 5 to 10), in Wiener's theory all the present and past values are needed for the prediction of the future. The solution of these diffeulties may be found in one or more of the following proiects:

(i) Device of an antomatic calculator.

(ii) Incorporation of various existing theories on meteorological phenomena for the determination of the constants of the system.

(iii) Formulation of some approximate procedure.

In the remaining part of this report will be described some consideration on the 3rd problem listed above.

\section{Decomposition of Time Series and the Application of Harmonic Analysis (8)}

Let the set of $n$ time series $q_{i}(t)^{\prime}$ 's be so chosen that one is possible to divide them into sets of functions $q_{1}(t), q_{2}(t), \ldots, q_{m}(t)$ and $q_{m+1}(t), q_{m+2}(t), \ldots, q_{n_{0}}(t)$, where the functions of each set belong to different frequency regions, so that using the formal Fourier transform of each time series

$$
q_{k}(t)=\int_{-\infty}^{\infty} f_{k}(\nu) e^{2 \pi i v t} d \nu, \quad k=1,2, \ldots, n,
$$

(7) M. Ogawara: Reports from the Central Met. Obs., No. 24, 1949; M. Ogawara and T. Fujita: Forecasting of Wolf's Sun-spot Numbers by Stochastic Extrapolation (unpublished).

(8) The simplest case of the prediction of a single time series is considered here. An extension to multiple time series would be trivial. 
one has

$$
f_{i}(\nu) f_{j}(\nu)=0, \quad\left\{\begin{array}{l}
i=1,2, \ldots, m, \\
j=m+1, \ldots, n .
\end{array}\right.
$$

In this case the time series belonging to different sets are statistically independent. In particular, one has

$$
\left[q_{i}(t+\tau) q_{j}(t)\right]_{i}=0, \quad\left\{\begin{array}{l}
i=1,2, \ldots, m, \\
j=m+1, \ldots, n .
\end{array}\right.
$$

It is further assumed, as is often practically the case, that the characteristic solutions

$$
\left.\begin{array}{rlrl}
z_{t} & =z_{\delta_{0}} e^{\lambda_{i} t}, & & \text { for } t>0 \\
& =0, & \text { for } t<0
\end{array} \quad i=1,2, \ldots, n\right\}
$$

have theri amplitude spectra falling entirely into either of the two frequency regions. Let $\lambda_{1}, \lambda_{2}, \ldots, \lambda_{m}$ belong to the first set and the remaining ones to the second. Then from (28) one has

$$
\begin{aligned}
& q_{i}(t)=\sum_{j=1}^{m} \tilde{c}_{i j} \int_{0}^{\infty} \pi j^{\prime}\left(t-t^{\prime}\right) e^{\lambda_{j} t^{\prime}} d t^{\prime}, \quad i=1,2, \ldots, m, \\
& =\sum_{j=m+1}^{n} \tilde{c}_{t j} \int_{0}^{\infty} \pi ;\left(t-t^{\prime}\right) e^{\lambda j t^{\prime}} d t^{\prime}, \quad i=m+1, \ldots, n,
\end{aligned}
$$

so that the Langevin equation will also be separated,

$$
\begin{aligned}
& \frac{d q_{i}}{d t}-\sum_{j=1}^{m} a_{i, q, j}^{\prime}=p_{i}^{\prime}(t), \quad i=1,2, \ldots, m, \\
& \frac{d q_{i}}{d t}-\sum_{j=m+1}^{n} a_{i, q}^{\prime}=p_{i}^{\prime}(t), \quad i=m+1, \ldots, n .
\end{aligned}
$$

Thus the problem is reduced to ones of lower order. This reduction may be carried on until the overlapping of the amplitude spectra of different characteristic solutions (34) violates the assumption used in the above argument. Even in the latter case one may '. proceed further under tolerable approximations. In favorable cases one would be possible to attain.complete separation of the different $\lambda_{i}$ 's by the above procedure without appreciable errors, so that it is finally reduced to a number of simplest types of Brownian motion.

At this point it would easily be recognized that the method of harmonic analysis plays an important role in the practical applications of the present theory. In the following will be described a formulation of the method which is intended to meet the requirements of the direct practical applications. First one starts with the observed time series $x(t)$ whose values are supposed to be known from a suffciently large negative value of time $-T$ to $t=0$. When the values of $x(t)$ are given for $a$ discrete set of times, one may either regard them to be substituted by a continuous curve which is obtained by the usual method of curve fitting, or replace various integrals in the formulation for continuous cases by proper summations in the discrete cases. As the nature of errors introduced by these modifications 
may be computed by the well-established method of Fourier integrals, it wlll be left out of consideration here.

The function $x(t)$ being given, one may easily calculate its Fourier transform

$$
A(\nu)=\int_{-T}^{0} x(t) e^{-2 \pi i \nu t} d t
$$

and its absolute magnitude $[A(\nu) \mid$. It must be noted here that the amplitude spectrum thus obtained contains a certain amount of indefiniteness in the sense that its fine structures in the irequency bands within a definite frequency difference $\Delta \nu=\mathbf{1} / T$ are physically meaningless. The amplitude spectrum given by $|A(\nu)|$ will usually consist of a number of maxima and minima showong more or less conspicuous predominancies in certain frequency bands, each one of which corresponding to one or more characteristic values discussed above. Owing to the above mentioned indeterminacy, however, only those maxima should be considered as significant ones whose adjacent frequency differences are appreciably greater than $1 / T$, so that. if there exists a definite upper bound in frequency - this is the case, for example, in discrete time series - the number of characteristic values becomes necessarily smaller than $2\left|\nu_{\max }\right| T$.

Now if some of the minima in the spectral curre are found to be negligibly small and almost touching the zero axis, one may safely take them as dividing points by which the characteristic solutions (34) are completely separated into a number of groups. As another extreme case one may consider the case in which two or more characteristic "resonance" frequencies overlap each other so that they fuse into a single maximum. The real situation is, however, that one does not know a priori how many resonance frequencies are in a given frequency band, but it is rather the spectral curve itself that gives any information about them. One "assumes" that a single maximum in the spectral curve corresponds to a single resonance frequency. The amount of error due to this assumption may be calculated in some special cases where some known functional forms are substituted in place of the experimental spectral curves.

The intermediate cases in which the adjacent maxima in the spectral curve are partly but not completely resolved, are those where most of the ambiguities occur. As an approximate procedure for these cases, one may take either of the following two ways: one assumes a single characteristic value $\lambda$ for a group of subsidiary maxima which as a whole constitute a single broader maximum; or one ascribes different values of characteristic solutions to each of the partly resolved maxima. It would be convenient to have some numerical criteria as to which one of the two alternatives should be taken, but the details will be treated in a subsequent paper.

It thus came to the conclution that in any case one can with different degrees of approximation reduce the problem into a set of harmonically bounded Brownian motions which include as a special case the motion of a free particle. Using real variables, the Langevin equations for them can be reduced to the form 


$$
\frac{d^{2} q}{d t^{2}}+\beta \frac{d q}{d t}+\omega_{0}^{2} q=p(t)
$$

or to

$$
\frac{d q}{d t}+\beta q=p(t)
$$

with

$$
\overline{p\left(t^{\prime}\right) p\left(t^{\prime \prime}\right)}=2 D \delta\left(t^{\prime}-t^{\prime \prime}\right)
$$

The solutions are well $k$ nown, ${ }^{(9)} i$. e, the conditional probability functions are Gaussian functions with the average and variance:
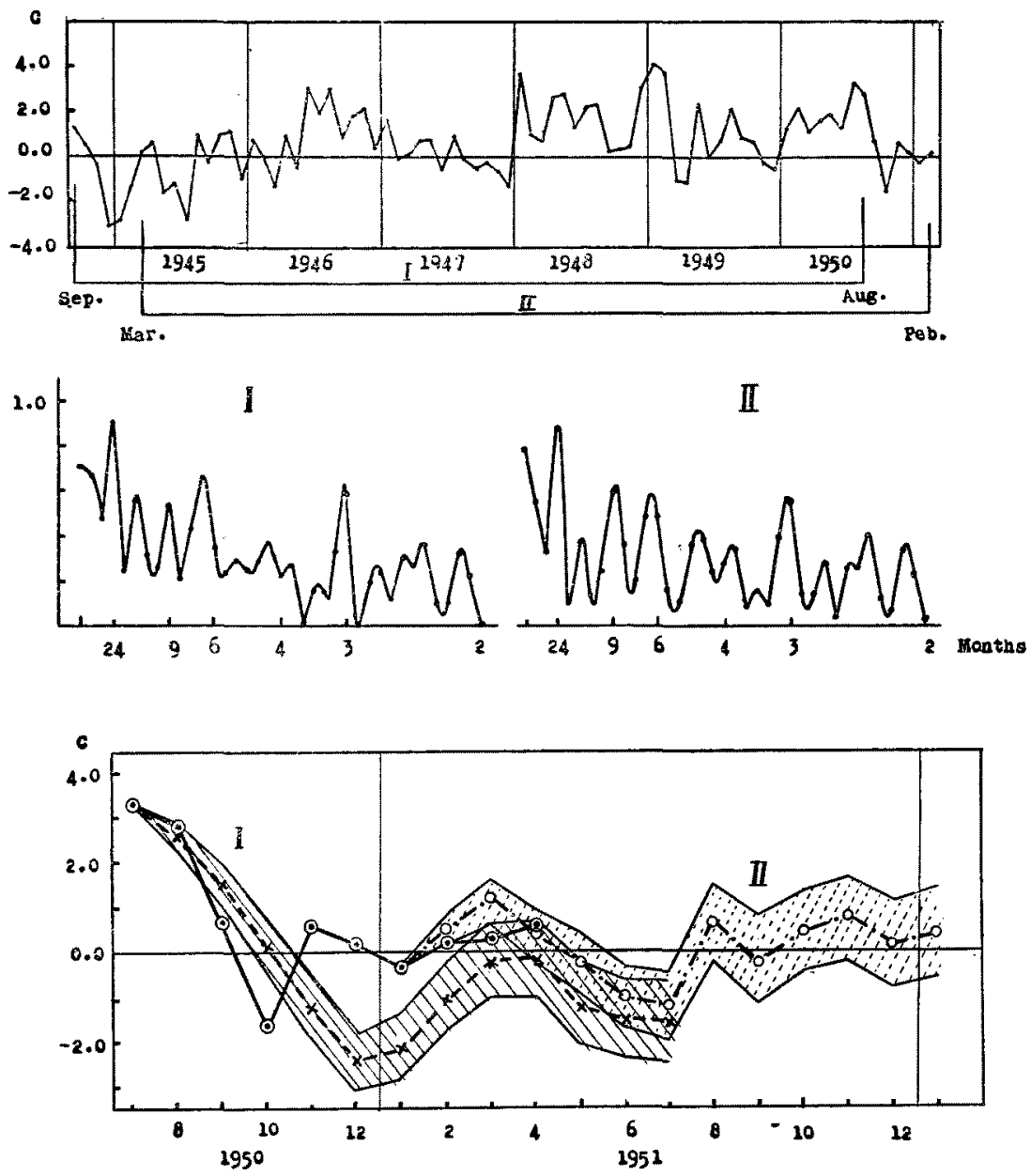

Fig. 1 a) Plot of monthly mean temperatures at Sapporo. b) Amplitude spectra for two different periods. c) Predicted values of monthly mean temperature at Sapporo. Observed values are shown by dotted circles.

(9) For example, Ming Chen Wang and G. E. Uhlenbeck, $l$. c.

$$
-11-
$$




$$
\begin{aligned}
\bar{q} & =\frac{\dot{q}_{0}}{\omega_{1}} e^{-\frac{1}{2} \beta t} \sin \omega_{1} t+\frac{q_{0}}{\omega_{1}} e^{-\frac{1}{2} \beta t}\left(\omega_{1} \cos \omega_{1} t+\frac{\beta}{2} \sin \omega_{1} t\right), \\
\overline{(q-\bar{q})^{2}} & =\frac{D}{\beta}\left[1-\frac{1}{\omega_{1}^{2}} e^{-\beta t}\left(\omega_{1}^{2}+\frac{1}{2} \beta^{2} \sin ^{2} \omega_{1} t-\beta \omega_{1} \sin \omega_{1} t \cdot \cos \omega_{1} t\right)\right], \\
\omega_{1}^{2} & =\omega_{0}^{2}-\beta^{2} / 4
\end{aligned}
$$

and

$$
\left.\begin{array}{rl}
\bar{q} & =q_{0} e^{-\digamma t}, \\
\overline{(q-\bar{q})^{2}} & =\frac{D}{\beta}\left[1-e^{-2 \beta t}\right],
\end{array}\right\}
$$

respectively for (36) and (37). Those for the original time series can be calculated by linear superposition of $\bar{q}$ 's and summing up the $\overline{(q-\bar{q})^{2}}$ 's thus obtained for different components.

In Fig. $1-3$ are shown a few examples of practical applications, which are intended
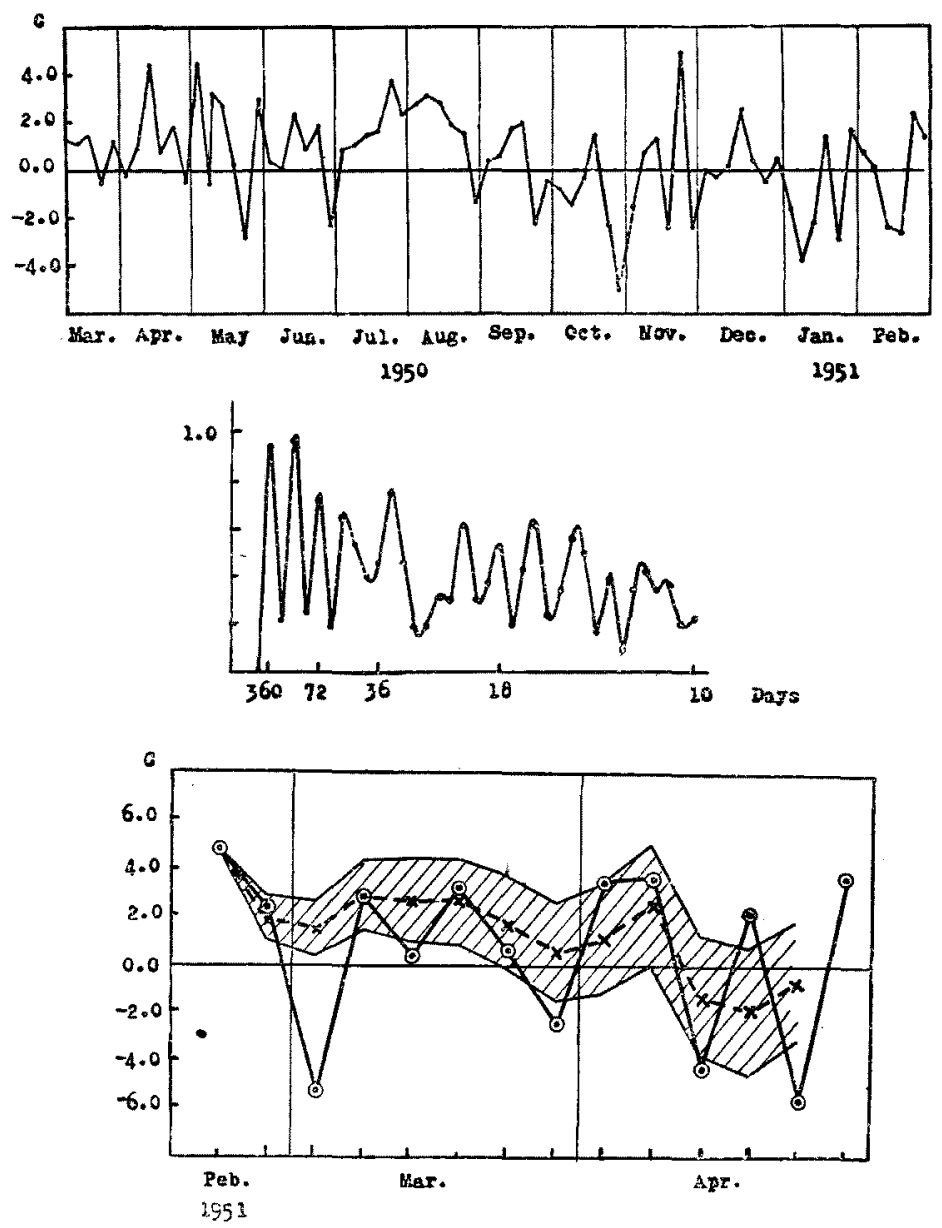

Fig. 2 a) Plot of mean temperatures of January at Sapporo. b) Amplitude spectrum. c) Predicted values for 1951. 

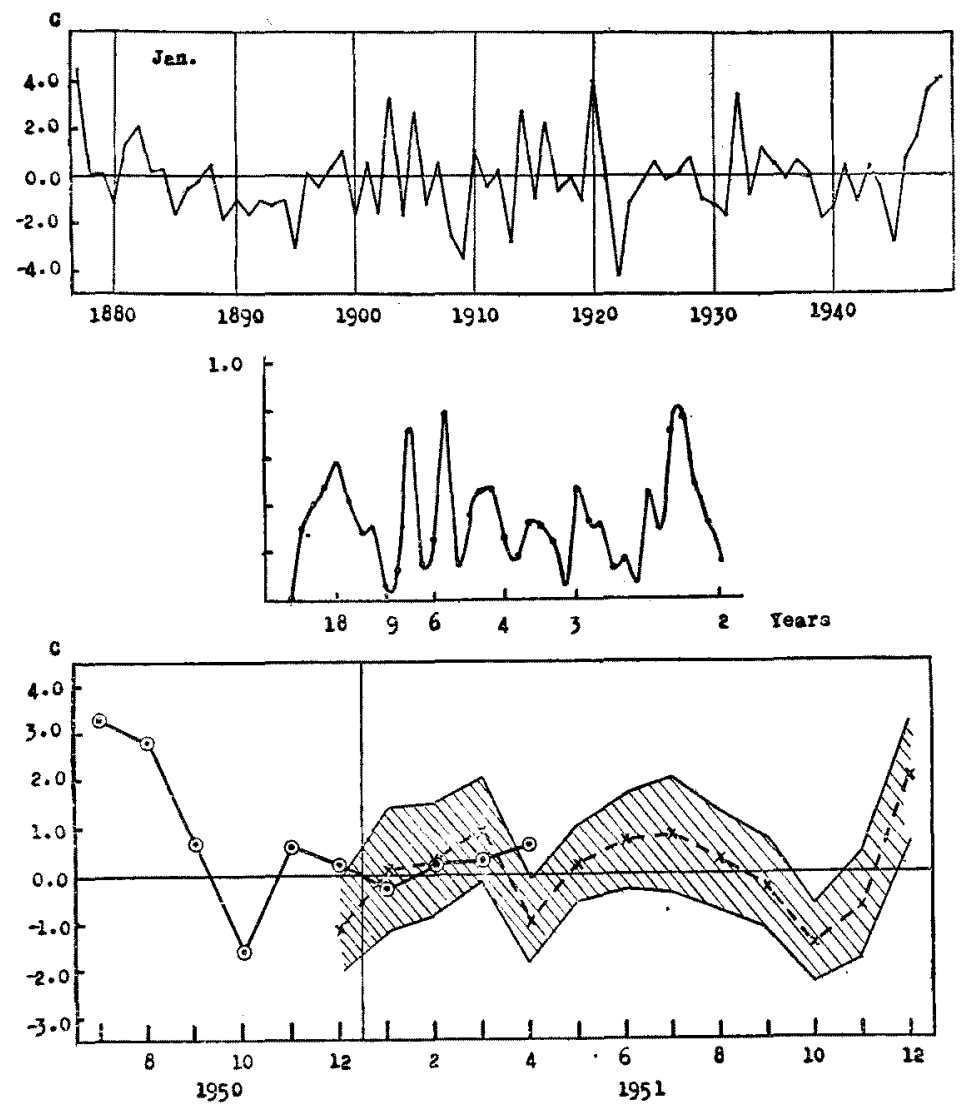

Fig. 3 a) Plot of 5-day mean temperatures at Sapporo. b) Amplitude spectrum. c) Predicted values.

not to be used as any routine works, but only to show how effective and promising the present method is. Fig. $1 \mathrm{a}$ is a plot of monthly mean temperatures at Sapporo from Sept. 1944 to Feb. 1951, expressed in deviations from the mean annual change. The amplitude spectra are shown in Fig. $1 \mathrm{~b}$ for two different periods $I$ and II. They were obtained by the usual method of harmonic analysis using 72 ordinates. The predicted values are given by Fig. $1 \mathrm{c}$, together with their probable errors shown by the shaded area. The dotted circles are the observed values. It would be interesting to note the approximate coincidence of the two predictions based upon different periods.

In Fig. 2 is shown another example of prediction of the monthly mean temperature obtained by a different procedure. In this case the 72 values of mean temperature of Jan. shown in Fig. 2 a were subjected to harmonic analysis, and the obtained spectrum-Fig. $2 \mathrm{~b}$ -was used to calculate the probabre temperature of Jan. in the future. The same procedure was repeated for Feb., etc., and the result is shown in Fig. $2 \mathrm{c}$. The agreement with obser-- 
vations-dotted circles-is fairly good. The last example is shown in Figs. $3 a, b, c$ for the case of prediction of the five-day mean temperatures obtained by similar calculations. While all these examples are based on the method of harmonic analysis using 72 ordinates, it would naturally be expected that better prediction should result by increasing the number of ordinates in the analysis.

In conclusion the authors wish to express their hearty thanks to Prof. T. Hori, the director of the Institute to which they belong, for the interest he have had in this work, and also to Dr. K. Takahashi and Mr. M. Ogawara, both of the Meteorological Research Institute, for the valuable discussions held about the present method. The data used for calculation in this paper were supplied by Mr. Y. Morita, of the Meteorological Observatory at Sapporo, to whom also the authors' appreciation should be expressed.

\title{
On the Turbulence in the Lower Troposphere
}

\author{
N. Saito \\ (Niigata Local Meteorological Obserratory)
}

\section{§ 1. Introduction}

There are eddies of various scales in the atmosphere, and the Austausch phenomena in the free air have been studied along two lines, one of which is the idea of Großturbulenz for large scale turbulences, and the other the theory of turbulence in the aero-and fluiddynamics for small scale turbulences.

The Großturbulenz has been studied by Defant, Lettau and Hillebrand, etc., and the eddy diffusion coefficient of the order of $10^{11} \mathrm{~cm}^{2} / \mathrm{sec}$ has been obtained by means of the average pressure pattern and the fluctuation of geostrophic wind.

The coefficient of large scale diffusion in the field of geostrophic wind seems to be relating to a phenomenon in the upper air where the surface friction is negligible, but in the lower troposphere, the friction of the earth's surface and the thermal disturbance are not negligible. So that, in the lower troposphere small tubulences may be found more irequently than large scale turbulences in the field of geostrophic wind. But the turbulence in the lower troposphere is of much larger scale than that in wind-tunnel experiments or the micro-oscillation of natural wind with the period of several seconds, and so, in this paper, such a turbulence in the lower troposphere is called a middle scale turbulence.

* The middle scale turbulence is found by analysing the meteorological phenomena in the lower troposphere, such as the oscillation of wind with the periods of $1 \sim 10$ minutes, the diffusion of volcanic ashes, and convective cells which appear in gusts of thunderstorm and bursts of precipitation in unstable currents, and may be regarded as eddies. 